\title{
A Rare Entity of Angioimmunoblastic T-Cell Lymphoma
}

\author{
Ganesh Kasinathan*, Ahlam Naila Kori \\ Haematology Unit, Tengku Ampuan Afzan Hospital, Kuantan, Pahang, Malaysia \\ Email: *ganeshkasinathan11@hotmail.com
}

How to cite this paper: Kasinathan, G. and Kori, A.N. (2019) A Rare Entity of Angioimmunoblastic T-Cell Lymphoma. Open Journal of Blood Diseases, 9, 41-46.

https://doi.org/10.4236/ojbd.2019.92005

Received: April 1, 2019

Accepted: June 7, 2019

Published: June 10, 2019

Copyright (C 2019 by author(s) and Scientific Research Publishing Inc. This work is licensed under the Creative Commons Attribution International License (CC BY 4.0).

http://creativecommons.org/licenses/by/4.0/

\begin{abstract}
Introduction: Angioimmunoblastic T Cell Lymphoma (AITL) is a well-recognized subtype of peripheral T cell lymphoma. It occurs predominantly in the lymph nodes with presence of systemic symptoms and carries a dismal prognosis. AITL accounts for about $1 \%-2 \%$ of all cases of non-Hodgkin lymphoma. Case presentation: A 59-year-old gentleman of Chinese ethnicity with no prior medical illness presented to Tengku Ampuan Afzan Hospital with a two-month history of fever, anorexia, unintentional weight loss and generalized lymphadenopathies. Physical examination revealed diffuse lymphadenopathies involving the cervical, axillary and inguinal regions bilaterally. $\mathrm{He}$ had hepatosplenomegaly. An excisional biopsy of the cervical and inguinal lymph nodes was compatible with AITL. The bone marrow biopsy demonstrated disease infiltration. He was treated with 6 cycles of Etoposide-CHOP (cyclophosphamide, doxorubicin, vincristine, prednisolone) induction chemotherapy followed by consolidation high dose therapy-autologous stem cell transplant (HDT-ASCT). He achieved complete remission on 18-Fluorodeoxyglucose Positron Emission Tomography (18-FDG-PET) imaging. His bone marrow biopsy showed disease clearance. Conclusion: The diagnosis of AITL remains challenging and often poses a dilemma to clinicians and lymphoma pathologists. The natural history of AITL remains very variable with many of them relapsing subsequently despite achieving prior complete remission.
\end{abstract}

\section{Keywords}

Angioimmunoblastic, Excision Biopsy, Etoposide-CHOP, Stem Cell Transplant

\section{Introduction}

Angioimmunoblastic T Cell Lymphoma (AITL) is a well-recognized subtype of 
peripheral T cell lymphoma. The revised World Health Organization (WHO) classification of lymphoid neoplasms 2016 characterizes AITL under the category of nodal T Cell lymphomas with $\mathrm{T}$ follicular helper phenotype [1]. AITL occurs predominantly in the lymph nodes with the presence of systemic symptoms and carries a dismal prognosis. AITL accounts for about $1 \%-2 \%$ of all cases of non Hodgkin lymphoma [2]. 20\% of cases of Peripheral T Cell Lymphoma (PTCL) are attributed to AITL annually [2]. The median age of diagnosis is 65 and AITL does not have a clear gender predisposition. The highest incidence rate of AITL is seen in Europe (28.7\%) [3]. Epstein Barr Virus is known to have a strong association with AITL with $80 \%$ - 95\% of biopsies revealing Epstein Barr Virus infection on in-situ hybridization (EBERish) [4]. Almost 70\% of patients with AITL have bone marrow involvement at diagnosis and most of them present at advanced stage [4]. The main objective of reporting this case is to share with the medical fraternity of a rare presentation of Angioimmunoblastic T Cell Lymphoma which has a variable natural history.

\section{Case Presentation}

A 59-year-old gentleman of Chinese ethnicity with no prior medical illness presented to Tengku Ampuan Afzan Hospital with a two-month history of fever, anorexia and unintentional weight loss. He complained of bilateral neck, axillary and inguinal swellings which were progressively enlarging over two months. $\mathrm{He}$ did not have any night sweats. There was no significant family history. $\mathrm{He}$ worked as an office clerk. He was a non-smoker and a teetotaler. He denied consuming any traditional or recreational drugs.

Physical examination revealed a gentleman with thin habitus. His ECOG (Eastern Cooperative Oncology Group) Performance Status was 1. He was febrile to touch. His blood pressure was $122 / 84 \mathrm{mmHg}$ with a pulse rate of 92 beats per minute. There were multiple palpable lymph nodes over the cervical, axillary and inguinal regions bilaterally measuring $3 \times 3 \mathrm{cms}$ in size the largest. His liver was palpable at $3 \mathrm{~cm}$ below the right costal margin with his spleen measuring $5 \mathrm{~cm}$ below the left costal margin. He did not have any skin lesions. Other systemic examinations were unremarkable.

His baseline staging whole body Computed Tomography (CT) imaging showed hepatosplenomegaly, hilar, bilateral cervical, axillary, intra-abdominal, pelvic, and bilateral inguinal lymphadenopathies. The largest lymph node measured $2.6 \mathrm{~cm}$.

An excision biopsy was performed. Cervical and inguinal lymph node histology (Figure 1 and Figure 2) showed strong intense positivity for pan $\mathrm{T}$ cell markers-CD2, CD3, CD4, CD5 with occasional CD 10 positivity and BCL-2 diffuse positivity for the lymphoid component. A total lack of CD20 positivity except for CD20 positivity highlighting the residual follicles with an enhanced dendritic framework by $\mathrm{CD} 23$ positivity was compatible with the diagnosis of AITL.

The staging bone marrow biopsy showed infiltrated marrow with fair reserve of haemopoietic activity. 


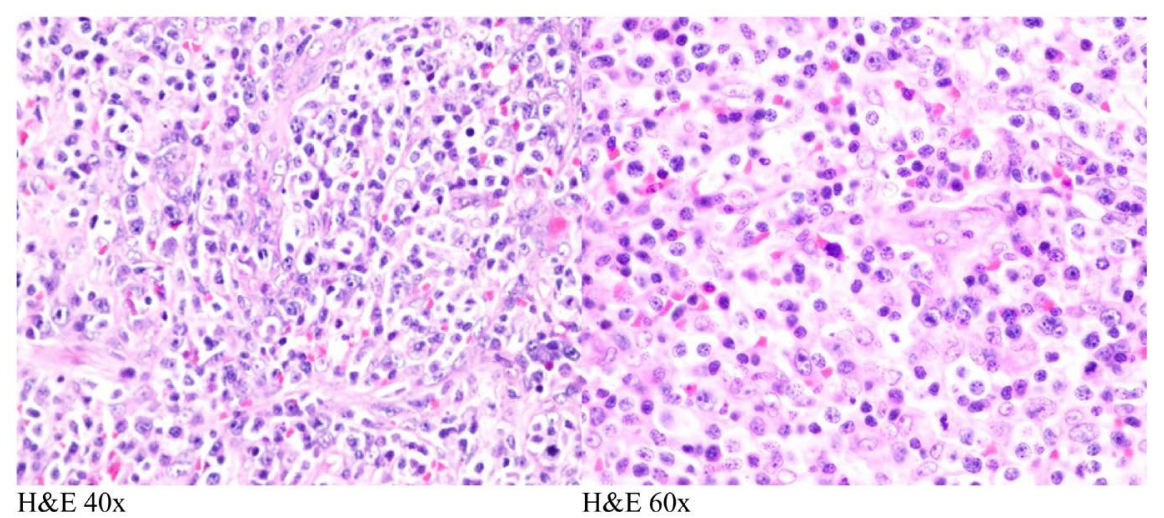

Figure 1. Haematoxylin \& Eosin (H\&E) shows sections of right cervical lymph nodes, multiple in nature, juxtaposed to each other, embedded within a fibrocollagenous and adipose framework. Individual lymph node with total effacement of lymph node architecture replaced by a diffuse distribution of small, intermediate and large malignant lymphoid cells with vesicular nuclei and prominent nucleoli within them is conspicuously observed. Occasional foci of faint follicular pattern is noticed towards the periphery.

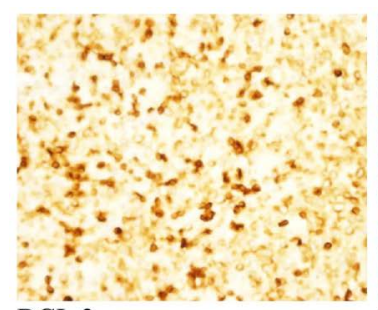

BCL 2

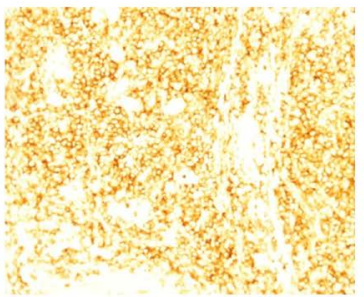

CD 4

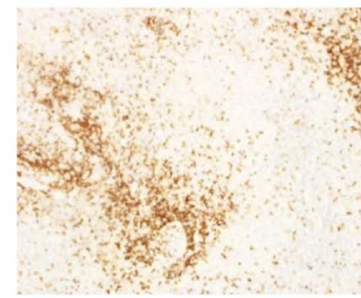

CD 20

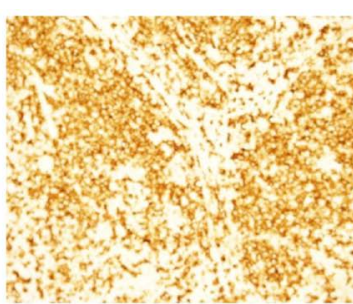

CD 2

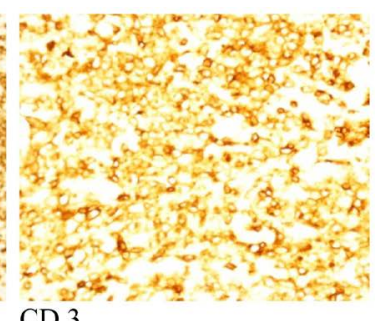

CD 3

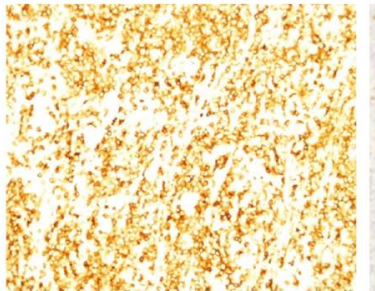

CD 5

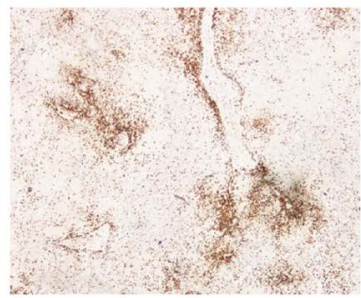

CD 20 (scanner image)
CD 10

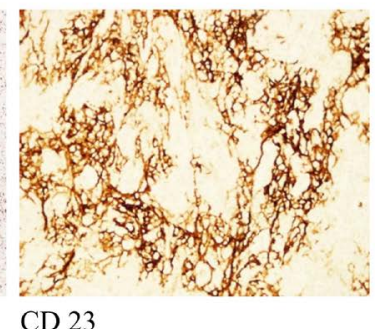

Figure 2. A panel of immunohistochemical staining ( $\times 20$ magnifications $)$ shows strong intense positivity for pan $\mathrm{T}$ cell markers-CD2, CD3, CD4, CD5 and BCL-2 diffuse positivity for the lymphoid component. A total lack of CD20 positivity except for CD20 positivity highlighting the residual follicles with an enhanced dendritic framework by CD23 positivity is compatible with the diagnosis of AITL.

There was no major paraprotein detected on serum and urine protein electrophoresis. 
His laboratory parameters are as tabulated below (Table 1).

A diagnosis of Stage IV Angioimmunoblastic T Cell Lymphoma, IPI (International Prognostic Index: score of 4-Hypoalbuminemia, advanced stage, elevated lactate dehydrogenase, more than 1 extranodal site involvement) was made. $\mathrm{He}$ was treated with 6 cycles of Etoposide-CHOP induction chemotherapy (Etoposide-Cyclophosphamide, doxorubicin, vincristine, prednisolone). Due to the natural history of the disease, he underwent upfront consolidation high dose therapy conditioning with BEAM (carmustine, etoposide, cytarabine, melphalan) followed by autologous stem cell transplant which was successful. The 18-fluorodeoxyglucose positron emission tomography imaging post autologous stem cell transplant showed complete metabolic response. His bone marrow biopsy demonstrated disease clearance. He is currently in complete remission for the past 16 months and is on follow up at our haematology clinic.

\section{Discussion}

AITL is a rare and aggressive T cell malignancy. The clinical symptoms are often vague and nonspecific such as seen in this case. Patients present with predominantly B symptoms such as prolonged fever, unintentional weight loss and night sweats. Lymphadenopathy is often generalized. 20\% - 50\% of AITL patients have skin rash which can vary from urticaria-like lesions to nodular tumours [5].

The gold standard for the diagnosis of AITL is an excisional lymph node biopsy. Most centers are inclined to perform core biopsies or fine needle aspirations as they save time and are less invasive. Frequently, core biopsies have small yields and are insufficient for adequate immunohistochemical analysis. In differentiating between malignant lymphoma and reactive lymph node hyperplasia,

Table 1. Laboratory Parameters.

\begin{tabular}{cc}
\hline Laboratory Parameters & Value (Normal Range with System International Unit) \\
\hline Haemoglobin & $10.7(13.5-16.5 \mathrm{~g} / \mathrm{dL})$ \\
Total White Cell Count & $6.6\left(4-10 \times 10^{9} / \mathrm{L}\right)$ \\
Platelet & $211\left(150-400 \times 10^{9} / \mathrm{L}\right)$ \\
Absolute Eosinophil count & $0.1\left(0-0.6 \times 10^{9} / \mathrm{L}\right)$ \\
Creatinine & $83(72-127 \mathrm{umol} / \mathrm{L})$ \\
Uric Acid & $3.9(3.5-7.2 \mathrm{mg} / \mathrm{dL})$ \\
Lactate Dehydrogenase & $530(100-190 \mathrm{U} / \mathrm{L})$ \\
Aspartate Aminotransferase & $36(0-40 \mathrm{U} / \mathrm{L})$ \\
Alanine Aminotransferase & $32(0-40 \mathrm{U} / \mathrm{L})$ \\
Albumin & $25(35-55 \mathrm{~g} / \mathrm{L})$ \\
Calcium & $2.2(2.2-2.6 \mathrm{mmol} / \mathrm{L})$ \\
HIV-1 and 2 serology & Non reactive \\
Epstein Barr Virus serology and PCR & Not detected \\
Direct Antiglobulin Test (DAT) & Negative
\end{tabular}


core biopsies revealed a high false negative rate and a low negative predictive value [6].

The cell of origin for AITL is the follicular helper T cells $\left(\mathrm{T}_{\mathrm{FH}}\right)$, an effector $\mathrm{T}$ Cell subset [7]. High power view microscopy of the lymph node demonstrates abundant immunoblasts, B cells, plasma cells, eosinophils, epithelioid cells, follicular dendritic cells and high endothelial venules [7].

Neoplastic T cells in AITL frequently express CXCL13, BCL-6, CD40L, CD 200 and ICOS with 95\% of AITL cases show enhanced expression of at least one of the following markers: SLAM-associated protein, programmed death-1 (PD-1) and c-Maf [8].

There is no gold standard chemotherapy for AITL. Anthracycline-based chemotherapy protocols such as $\mathrm{CHOP}$ (cyclophosphamide, doxorubicin, vincristine, prednisolone) appears to be the frontline regimen in many centers but the outcomes are often disappointing. CHOP carries a complete response (CR) rate of $39 \%$ in an upfront setting and specifically in AITL, a 53\% CR rate [9]. The addition of etoposide to CHOP in AITL demonstrates promise as an induction regimen with high overall response rates (ORR) at $82 \%$ and a CR rate of $51 \%$ [10]. High dose therapy followed by autologous stem cell transplant as upfront consolidation has become an increasingly used modality in those who are chemosensitive [10]. A large retrospective analysis from the European Group for Blood and Marrow Transplantation (EBMT) reported an improved response rate of $76 \%$ and a 5 -year OS of $44 \%$ in AITL.

\section{Conclusion}

The diagnosis of AITL remains challenging and often poses a dilemma to clinicians and lymphoma pathologists. Obtaining an excision biopsy often provides good yield thus hastening the diagnosis of AITL. The natural history of AITL remains very variable and carries a poor prognosis with many of them relapsing subsequently despite achieving prior complete remission.

\section{Consent}

Consent has been obtained from the patient for publication.

\section{Conflicts of Interest}

The authors declare no conflicts of interest regarding the publication of this paper.

\section{References}

[1] Swerdlow, S.H., Campo, E., Pileri, S.A., et al. (2016) The 2016 Revision of the World Health Organization Classification of Lymphoid Neoplasms. Blood, 127, 2375-2390. https://doi.org/10.1182/blood-2016-01-643569

[2] Frizzer, A.G., Moran, E.M. and Rappaport, H. (1975) Angio-Immunoblastic Lymphadenopathy: Diagnosis and Clinical Course. The American Journal of Medicine, 59, 803-818. https://doi.org/10.1016/0002-9343(75)90466-0 
[3] Federico, M., Rudiger, T., Bellei, M., et al. (2013) Clinicopathologic Characteristics of Angioimmunoblastic T-Cell Lymphoma: Analysis of the International Peripheral T-Cell Lymphoma Project. Journal of Clinical Oncology, 31, 240-246. https://doi.org/10.1200/JCO.2011.37.3647

[4] Balagué, O., Martínez, A., Colomo, L., et al. (2007) Epstein-Barr Virus Negative Clonal Plasma Cell Proliferations and Lymphomas in Peripheral T-Cell Lymphomas: A Phenomenon with Distinctive Clinicopathologic Features. The American Journal of Surgical Pathology, 31, 1310-1322.

https://doi.org/10.1097/PAS.0b013e3180339f18

[5] Siegert, W., Nerl, C., Agthe, A., et al. (1995) Angioimmunoblastic lymphadenopathy (AILD)-Type T-Cell Lymphoma: Prognostic Impact of Clinical Observations Andlaboratory Findings at Presentation. Annals of Oncology, 6, 659-664.

https://doi.org/10.1093/oxfordjournals.annonc.a059281

[6] Ben-Yehuda, D., Polliack, A., Okon, E., et al. (1996) Image-Guided Core Needle Biopsy in Malignant Lymphoma: Experience with 100 Patients That Suggests the Technique Is Reliable. Journal of Clinical Oncology, 14, 2431-2434. https://doi.org/10.1200/JCO.1996.14.9.2431

[7] Willenbrock, K., Brauninger, A. and Hansmann, M.L. (2007) Frequent Occurrence of B-Cell Lymphomas in Angioimmunoblastic T-Cell Lymphoma and Proliferation of Epstein-Barr Virus-Infected Cells in Early Cases. British Journal of Haematology, 138, 733-739. https://doi.org/10.1111/j.1365-2141.2007.06725.x

[8] Iannitto, E., Ferreri, A.J. and Minardi, V. (2008) Angioimmunoblastic T-cell lymphoma. Critical Reviews in Oncology/ Hematology, 68, 264-271. https://doi.org/10.1016/j.critrevonc.2008.06.012

[9] Simon, A., Peoch, M., Casassus, P., Deconinck, E., et al. (2010) Upfront VIP-Reinforced-ABVD (VIP-rABVD) Is Not Superior to CHOP/21 in Newly Diagnosed Peripheral T-Cell Lymphoma. Results of the Randomized Phase III Trial GOELAMS-LTP95. British Journal of Haematology, 151, 159-166. https://doi.org/10.1111/j.1365-2141.2010.08329.x

[10] D’Amore, F., Relander, T., Lauritzsen, G.F., et al. (2012) Up-Front Autologous Stem-Cell Transplantation in Peripheral T-Cell Lymphoma: NLG-T-01. Journal of Clinical Oncology, 30, 3093-3099. https://doi.org/10.1200/JCO.2011.40.2719 